\title{
Effect of an external electric field on capillary filling of water in hydrophilic silica nanochannels
}

Karna, Nabin Kumar; Rojano Crisson, Andres; Wagemann, Enrique; Walther, Jens H.; Zambrano, Harvey A.

Published in:

Physical Chemistry Chemical Physics

Link to article, DOI:

10.1039/C8CP03186J

Publication date:

2018

Document Version

Peer reviewed version

Link back to DTU Orbit

Citation (APA):

Karna, N. K., Rojano Crisson, A., Wagemann, E., Walther, J. H., \& Zambrano, H. A. (2018). Effect of an external electric field on capillary filling of water in hydrophilic silica nanochannels. Physical Chemistry Chemical Physics, 20, 18262-18270. https://doi.org/10.1039/C8CP03186J

\section{General rights}

Copyright and moral rights for the publications made accessible in the public portal are retained by the authors and/or other copyright owners and it is a condition of accessing publications that users recognise and abide by the legal requirements associated with these rights.

- Users may download and print one copy of any publication from the public portal for the purpose of private study or research.

- You may not further distribute the material or use it for any profit-making activity or commercial gain

- You may freely distribute the URL identifying the publication in the public portal 

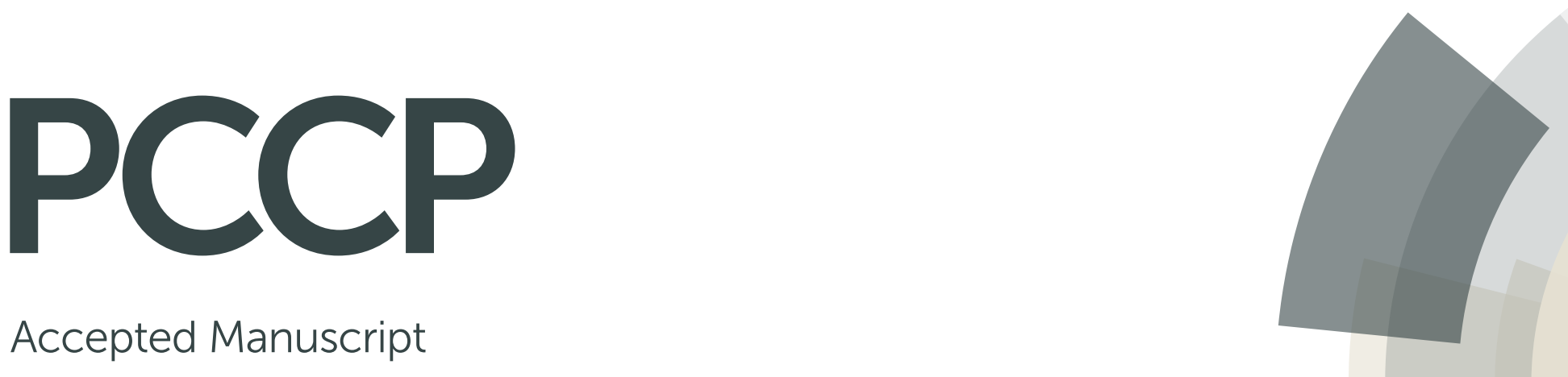

\section{Accepted Manuscript}

This article can be cited before page numbers have been issued, to do this please use: N. K. Karna, A. E. Rojano, E. I. Wagemann, J. H. Walther and H. A. Zambrano, Phys. Chem. Chem. Phys., 2018, DOI:

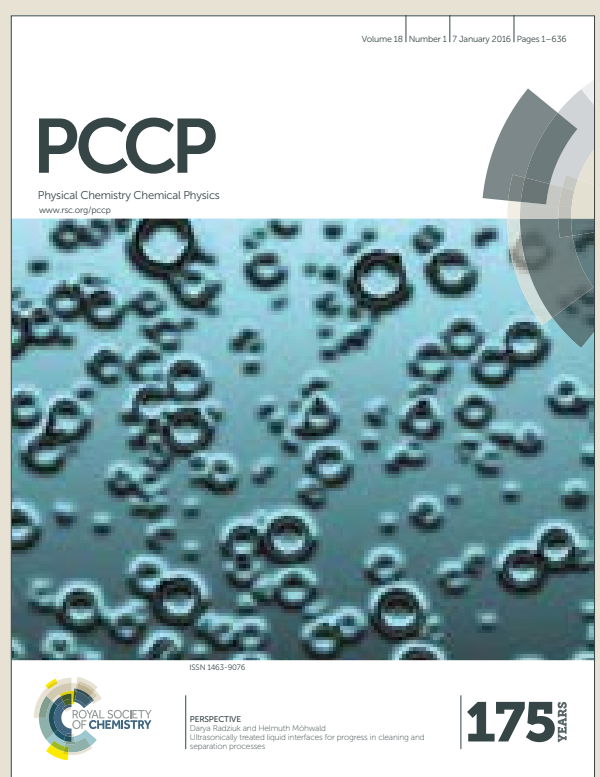

This is an Accepted Manuscript, which has been through the Royal Society of Chemistry peer review process and has been accepted for publication.

Accepted Manuscripts are published online shortly after acceptance, before technical editing, formatting and proof reading. Using this free service, authors can make their results available to the community, in citable form, before we publish the edited article. We will replace this Accepted Manuscript with the edited and formatted Advance Article as soon as it is available.

You can find more information about Accepted Manuscripts in the author guidelines.

Please note that technical editing may introduce minor changes to the text and/or graphics, which may alter content. The journal's standard Terms \& Conditions and the ethical guidelines, outlined in our author and reviewer resource centre, still apply. In no event shall the Royal Society of Chemistry be held responsible for any errors or omissions in this Accepted Manuscript or any consequences arising from the use of any information it contains. 


\title{
Journal Name
}

\section{ARTICLE TYPE}

Cite this: DOI: $10.1039 / \operatorname{xxxxxxxxxx}$

\section{Effect of an external electric field on capillary filling of water in hydrophilic silica nanochannels ${ }^{\dagger}$}

\author{
Nabin Kumar Karna, ${ }^{a, b}$ Andres Rojano Crisson, ${ }^{a}$ Enrique Wagemann, ${ }^{a}$ Jens $\mathrm{H}$. \\ Walther ${ }^{c, d}$, Harvey A. Zambrano*e
}

Received Date

Accepted Date

DOI: $10.1039 / x x x x x x x x x x$

www.rsc.org/journalname

Development of functional nanofluidic devices requires understanding the fundamentals of capillary driven flow in nanochannels. In this context, we conduct molecular dynamics simulations of water capillary imbibition in silica nanoslits under externally applied electric $(E)$ fields with strengths between 0 to $1 \mathrm{~V} / \mathrm{nm}$. For increasing $E$-fields, we observe a systematic lowering in the meniscus contact angle and a decrease in the corresponding water filling rates. These results contrast markedly the classical WashburnBosanquet's equation which predicts an increase in filling rates for lower water contact angles. Our study provides evidence that the observed decrease in water filling rates can be attributed to the interplay between two underlying mechanisms, a reduced fluidity of interfacial water and a systematic alignment of the water molecules in the bulk as a response to the particular strength of the applied $E$-field. Therefore, during water capillary filling a constant $E$-field applied in the direction parallel to the water imbibition leads to a lower than expected filling rate caused by a viscosity increase in the bulk and an altered solid-liquid friction on the channel walls. These coupled mechanisms governing capillarity under the action of applied $E$ fields could be manipulated for controlling imbibition of polar liquid solutions in nanofluidic devices.

\footnotetext{
${ }^{a}$ Department of Chemical Engineering, Universidad de Concepcion, Concepcion, Chile.

${ }^{b}$ Technology Development Unit, Coronel, Chile.

${ }^{c}$ Technical University of Denmark, Copenhagen, Denmark.

${ }^{d}$ Chair of Computational Science, ETH Zurich, Zurich, Switzerland.

e Department of Mechanical Engineering, Universidad Tecnica Federico Santa Maria, Valparaiso, Chile. Tel: 56-32-2654561; E-mail: harvey.zambrano@usm.cl
}

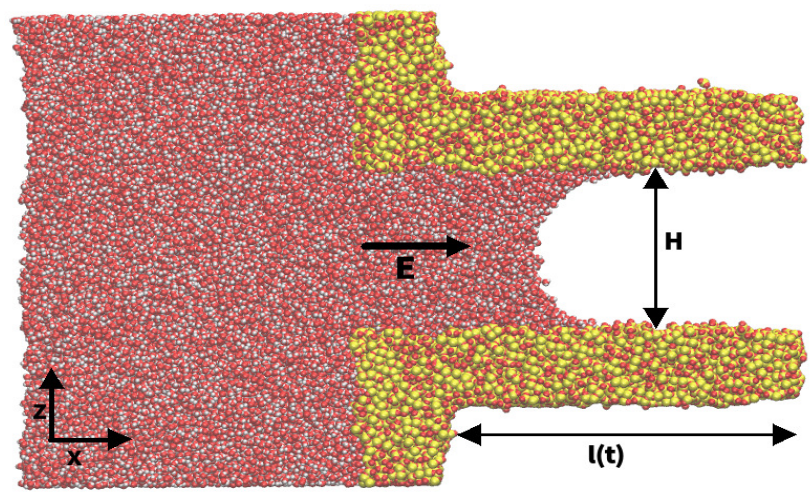

Fig. $1 \mathrm{~A}$ snapshot of capillary filling for 7-nm silica nanochannel. The electric field ( $E$ is applied in the direction of nanocapillary imbibition.

\section{Introduction}

Fabrication techniques have evolved dramatically over the last decade ${ }^{1-3}$ making possible to build artificial liquid conduits on length scales comparable to the size of ultraefficient biological channels. ${ }^{4}$ This realization offers new opportunities in the development of functional multistage microdevices integrated by nanoscale pores and channels ${ }^{5}$ which may have tremendous implications in a number of foreseeable biological, ${ }^{6}$ chemical $^{7}$ and technological applications. ${ }^{8}$ In fact, recent advances in nanofluidics have triggered a number of new high impact technologies such as high throughput techniques, ${ }^{9}$ drug delivery ${ }^{10}$ and biomolecular analysis. ${ }^{11}$

Water is an essential material in our everyday life and is the most used solvent in technological applications. ${ }^{12}$ Moreover, water in nanoconfinement plays a key role in the controlled transport of species through biological 
cellular membranes, ${ }^{4}$ in water desalination systems ${ }^{13}$ and in novel sensor technologies. ${ }^{14}$ Therefore, achieving precise control of water imbibition and transport in nanoscale structures is crucial to enable the design of more efficient processes and to develop a new generation of promising microdevices. Nevertheless, there are a number of challenges and opportunities associated with controlling water flow in nanochannels. Several studies have shown that water in nanoconfined geometries exhibits unexpected behaviors as compared to those observed in macroscale systems. ${ }^{15-19}$ For instance, the presence of interfaces distorts the near H-bond network of water and as a result of the ultra high surface to volume ratio inherent to nanoconfinement, altered interfacial properties greatly influence the static and dynamic behavior of water in nanochannels. ${ }^{2,20-23}$ In addition, water molecules are highly polar and hence, molecular ordering of water within ultraconfined structures can be altered by exposure to electromagnetic fields. ${ }^{23-25}$ In this context, a variety of techniques like direct modification of the surface charge density, ${ }^{26,27}$, tuning the solid-liquid interfacial tension by applying electric potentials ${ }^{28}$, field effects induced by surface embedded electrodes ${ }^{29,30}$ and electric, ${ }^{24,31,32}$ and magnetic far field effects ${ }^{33}$ have been used for manipulation of aqueous solutions in nanoconfinement. Furthermore, recent studies have revealed the significant effect of varying external applied electric fields on flow control by tuning the electrical double layer thickness, ${ }^{29,34}$ the electro-osmotic flow velocity ${ }^{35-37}$ and the pressurized water flow rates. ${ }^{38}$.

In the present study, we analyze the effect of an externally applied electric field on capillary flow of water in hydrophilic silica nanochannels cf. figure 1). It is well established that the driving force in capillary flow is determined by a balance between the Laplace pressure ${ }^{39,40}$ and a drag with variable contributions from inertia and viscous friction. ${ }^{41,42}$ Indeed, the Laplace pressure is directly related to the surface tension acting on the curved interface at the advancing capillary front whose curvature in turn is dependent on the surface wettability of the channel walls. Furthermore, wettability is quantified by measuring the water contact angle which is mainly determined by the particular strength of the solid-liquid interactions. ${ }^{32,43,44}$ In previous studies, we reported the relation between dynamic contact angle, height of nanochannels and capillary filling flow rates. ${ }^{42,45}$ Specifically, we found that the initial filling rate depends upon the particular value of the contact angle made by the fluid at the entrance of the nanochannel, and is given by:

$$
U_{i}=\sqrt{\left(\frac{2 \gamma \cos \theta_{i}}{\rho H}\right)}
$$

where, $U_{i}$ corresponds to the initial flow velocity and $\theta_{i}$ is the initial value of the contact angle made by fluid at the channel entrance.

In capillary filling under the influence of an externally applied electric field, the meniscus contact angle of the imbibing fluid can be predicted by the Young-Lipmann (Y-L) equation 46,47

$$
\cos \theta=\frac{\gamma_{s g}-\gamma_{s l}}{\gamma_{l g}}+\frac{\left\langle\varepsilon_{0} \varepsilon|E|^{2} D\right\rangle}{2 \gamma_{l g}}
$$

where $\theta$ is the equilibrium contact angle, $D$, the thickness of the solid-liquid interfacial layers, $\gamma$, the surface tension of the liquid, $E$, the applied electric field, $\varepsilon$, the dielectric constant of the liquid and $\varepsilon_{0}$, the permittivity of free space. The subscripts $l, g$ and $s$ refer to the liquid, gas and solid, respectively, and the angular bracket stands for the average over $D$. The average interaction between a free dipole $(\mu)$ and electric field $(E)$ at temperature $T$ is $\sim|\mu|^{2}|E|^{2} /\left(3 k_{B} T\right)^{47}$, where $k_{B}$ represents the Boltzmann's constant. The Y-L equation indicates that contact angle depends only on the absolute strength of the field regardless of its direction. However, deviations from these predictions have been reported in studies of water contact angles at hydrophobic surfaces under different electric field strengths at the nanoscale. ${ }^{47-50}$ Qualitatively, the Y-L equation indicates lowering of contact angle for increasing applied electric field and hence predicts, at first glance, higher capillary pressure with increase in electric field strength during imbibition in hydrophilic nanochannels. Nevertheless, the application of an electric field during the capillary filling of water in nanochannels could affect not only the meniscus contact angle ${ }^{49}$ but also other properties such as viscosity, ${ }^{51}$ dielectric constant, ${ }^{52}$ temperature and surface tension $^{53}$ among others ${ }^{38,51}$ and therefore, it might be challenging to predict the effect of an electric field applied during the water imbibition process at nanoscale.

In this work, from large scale molecular dynamics simulations of water imbibition in the presence of an electric field applied in the direction parallel to the flow, we report the time evolution of the capillary front position and the instantaneous meniscus contact angle during the capillary filling of a $7 \mathrm{~nm}$ high nanoslit. The strengths of the electric fields applied to the system range from 0 to $1 \mathrm{~V} / \mathrm{nm}$. These field strengths are in the range of those produced by charged electrodes, ${ }^{54}$ biological ion channels, ${ }^{55}$ electricfield-directed assembly of biomolecules ${ }^{56}$ and electropora- 
tion processes. ${ }^{57}$ It should be noted that in experiments the effective electric field differs significantly from the applied field because the actual field is lowered by the orientational polarization of water. ${ }^{49,58}$ Therefore, in the bulk phase, the exact relation (for the absolute values of the field) is given by 59

$$
E=\frac{3 E_{0}}{\varepsilon_{r}+2}
$$

where $E_{0}$ is the applied electric field, and $\varepsilon_{r}$ is the relative permittivity, defined as the ratio of effective permittivity of the medium $(\varepsilon)$ to the absolute permittivity of free space $\left(\varepsilon_{0}\right)$. Moreover, the strength of the electric field used in the present study are weak enough to avoid decomposition of water molecules. ${ }^{60}$ In our simulations, the application of an electric field exerts a force $F=q_{i} E$ at the center of mass of each atom according to the partial charge $q_{i}$ on each corresponding atom which is discussed in detail elsewhere. ${ }^{29,49,58}$ As water molecules are electrically neutral but highly polarized, the effect of an external electric field on water is a torque which reorients each molecule in the direction of the applied field altering the overall hydrogen bonding network. ${ }^{38,61}$ Furthermore, under the influence of an increasing external electric field, water molecules may experience progressive reduction in the degrees of freedom. ${ }^{23,44,62}$

\section{Computational method}

To elucidate the effect of externally applied electric fields in capillary filling of water in hydrophilic silica nanopores, we conduct a series of all-atom MD simulations using FASTTUBE, a molecular dynamics simulation package which has widely been used to investigate fundamental fluid dynamics under nanoconfinement. ${ }^{29,42,45,63-65}$ Water molecules are described using the simple point charge SPC/E model ${ }^{66}$ and the silica atoms by the TTAMm model developed by Guissani and Guillot ${ }^{67}$ which is a modification of the original TTAM model. ${ }^{68}$ Water-silica interactions are described using Coulomb and Buckingham potentials calibrated in our previous study ${ }^{69}$ wherein the experimental value of the water contact angle reported by Thamdrup et al. ${ }^{70}$ in studies of capillary filling is used as criterion to calibrate the potential parameters. For further details of the potentials used here, we refer readers to Zambrano et al. ${ }^{69} \mathrm{We}$ have chosen the SPC/E water model not only to be consistent with our previous works, ${ }^{42,45,63,69}$ but also due to its simplicity and quantitative agreement with experiments to yield water reorientation and hydrogen bond dynamics. ${ }^{44,51,71-73}$ Moreover, studies have found that the value of the surface tension for the SPC/E model is in good agreement with experimental results. ${ }^{74}$

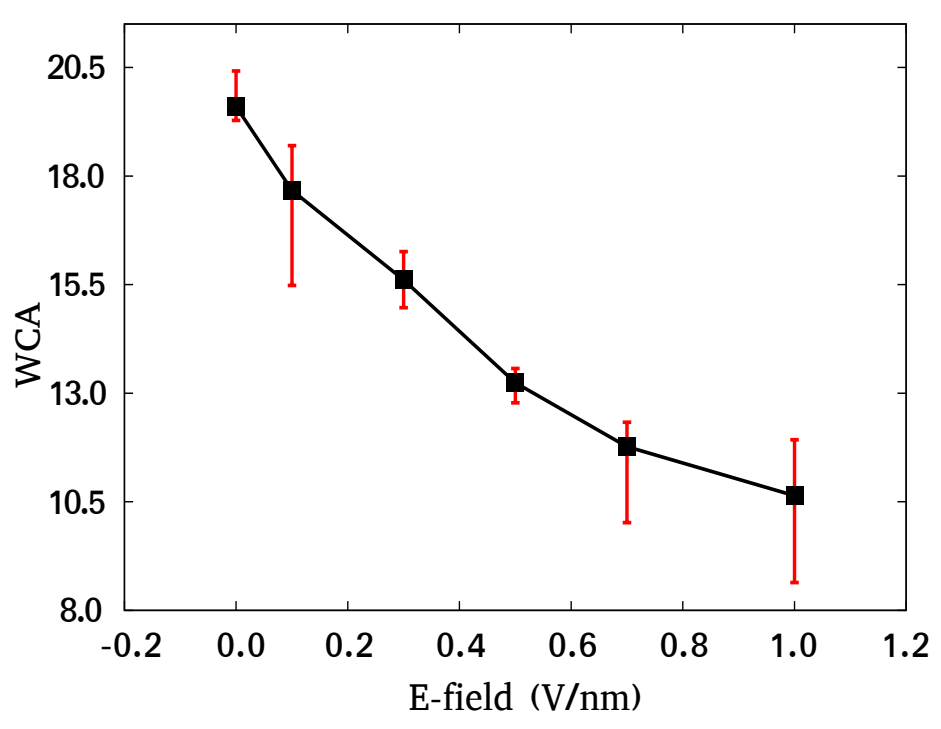

Fig. 2 Water contact angle (WCA) on a hydrophilic silica slab at different electric field strength. The electric field is applied parallel to the surface.

\section{Results and discussion}

\section{Influence of electric field in wettability}

We first study the effect of electric field strength on water wettability of a silica slab by evaluating the water contact angle (WCA) on a hydrophilic amorphous silica surface. For this purpose, we perform simulations of a cylindrical nanodroplet, ${ }^{75}$ consisting of 3000 water molecules mounted on a silica slab of $37.92 \mathrm{~nm} \times 2.584 \mathrm{~nm} \times 3.5 \mathrm{~nm}$ in vacuum using a time step of $2 \mathrm{fs}$. To equilibrate the water droplet-silica system, we follow the methodology described by Zambrano et al. ${ }^{69}$ and references therein. Further, we allow a cylindrical water droplet to spread on a frozen silica slab under different electric field strengths with the field applied parallel to the solid surface. A Berendsen thermostat $^{76}$ is connected to the water molecules with a weak coupling constant of $0.1 \mathrm{ps}$ to maintain a constant temperature of $300 \mathrm{~K}$. We use the Berendsen heat bath to control the system temperature as this thermostat may have advantages over other methods used in nonequilibrium simulations of viscous flow. ${ }^{38,77}$ The equilibrium value of water contact angles are obtained as the average value for snapshots extracted every 50 time steps from trajectories computed after $5 \mathrm{~ns}$ of equilibration. ${ }^{48,49}$ The values of the water contact angles on the silica surface in either the absence or presence of externally applied fields are plotted in figure 2. The equilibrium WCA in absence of electric field is measured to be ca. $20^{\circ}$ which is in accordance with the value previously reported. ${ }^{69}$ Meanwhile, a significant decrease in WCA can be observed with increasing electric field strength, which indicates that the magnitude of the 


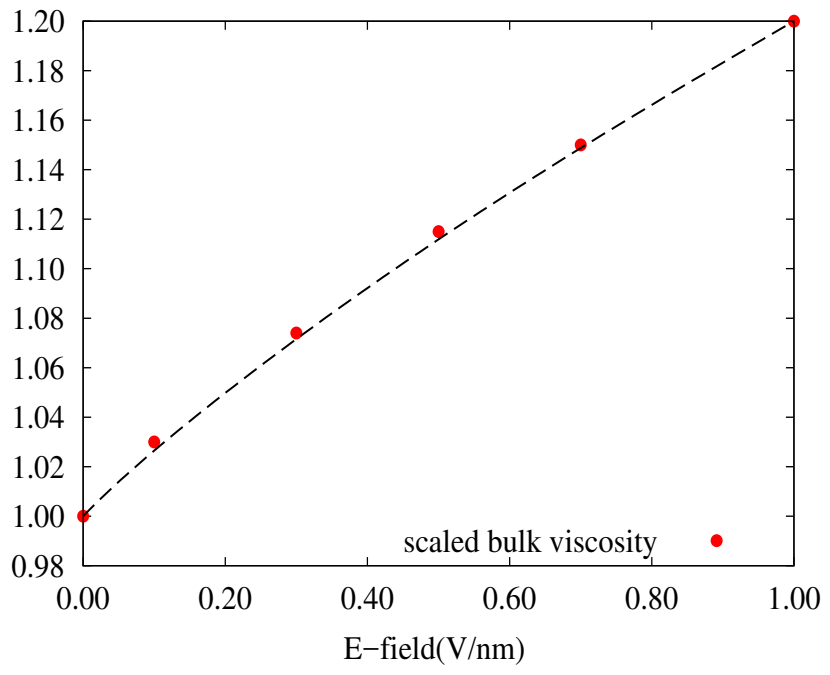

Fig. 3 Scaled viscosity calculated from the Periodic perturbation method at different electric fields. The dotted lines represent the linear tendency of the change in viscosity while the points are the measured values.

applied electric field has a strong effect in the final equilibrium state of the droplet. ${ }^{49,49,72}$

\section{Influence of electric field in the bulk viscosity of water}

In the present study, we calculate the viscosity of bulk water using the periodic-perturbation method developed by Hess, ${ }^{78}$ where the viscosity of liquid water is calculated by applying an external force on each water molecule. The applied force is a function of height $(z)$ of the simulation box. For details of the viscosity calculation using this method, readers are referred to the studies by Hess ${ }^{78}$ and Sunda et al. ${ }^{79}$ We plot the scaled viscosity $\left(\mu_{s}\right)$ of bulk water at different field strengths as shown in figure 3 , where,

$$
\mu_{s}=\frac{\mu_{f}}{\mu_{0}}
$$

$\mu_{f}$ and $\mu_{0}$ represent the bulk viscosities of water, with and without imposed electric field respectively. Within the range of electric field considered in this study, the viscosity increases linearly with increasing field strength. The maximum change in viscosity between the cases with the maximum applied field and without electric field respectively is found to be ca. $20 \%$. These results are in line with those reported by Zong et al. ${ }^{51}$ using non-equilibrium molecular dynamics, who observed that the dipole moment of the water molecules in bulk align along the direction of the applied electric field resulting in a monotonic increase of the bulk viscosity of water with electric field strength.

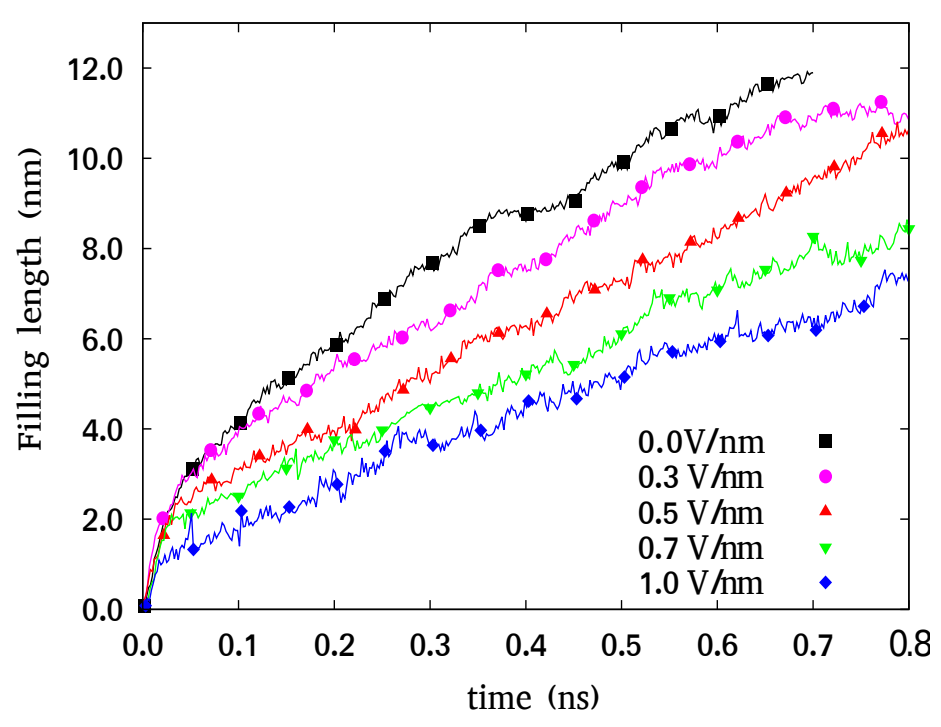

Fig. 4 Nanocapillary imbibition length as a function of time in a $7 \mathrm{~nm}$-high channel for different strength of electric field. The constant filling rates for all the cases of electric field indicates an inertial regime independent of initial conditions.

Effect of electric field on capillary imbibition in silica nanochannels

To study the capillary imbibition of water in silica nanochannels in the presence of an external electric field we build a channel with height of $7 \mathrm{~nm}$ using parallel walls consisting of amorphous silica slabs as shown in figure 1 . For the details of the slab construction and amorphization, readers are referred to our previous studies. ${ }^{45,69}$ Simulations are conducted in a periodic orthorhombic cell with size of $2.52 \mathrm{~nm} \times 43.0 \mathrm{~nm} \times 20.88$ along the $x, y$ and $z$ axis. Part of the simulation box is filled with 23,600 water molecules to reproduce the density of water in bulk. In all the simulations of imbibition, the water molecules are coupled to a Berendsen ${ }^{76}$ heat bath with a coupling constant of $0.1 \mathrm{ps}$ at $300 \mathrm{~K}$ during $1 \mathrm{~ns}$; then, the thermostat is disconnected and the simulations are conducted in the microcanonical ensemble (NVE) for 3 ns until the system is equilibrated. Next, the electric field is applied and the system is further allowed to relax for $1 \mathrm{~ns}$ after which the water slab is released from rest to move spontaneously towards the silica channels. We notice that the electric fields are imposed, for all the cases, in the direction parallel to the water capillary imbibition.

The viscous Joule heat is extracted during the filling connecting a Berendsen thermostat to the water molecules with a weak coupling constant of 0.1 ps while atoms of the channel walls are fixed to their equilibrium positions. ${ }^{58}$ In particular, the use of a Berendsen heat bath to control temperature could not be ideal ${ }^{58}$ however previous studies have reported, for water under electric fields, satisfactory 


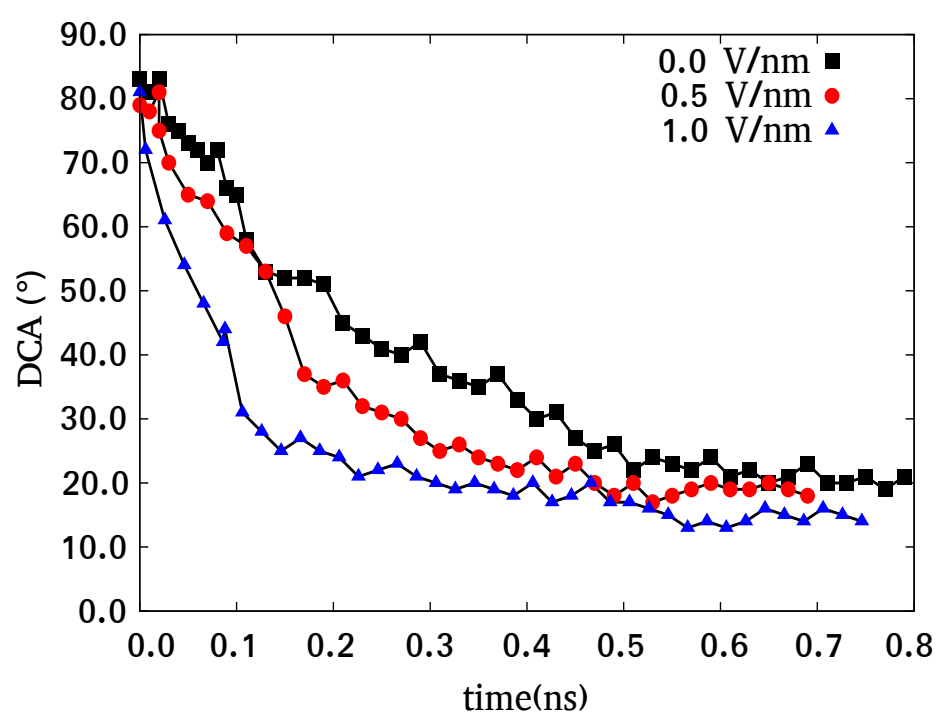

Fig. 5 Measured dynamic contact angles in a $7 \mathrm{~nm}$-high channel at various electric field strengths.

results using a Berendsen thermostat with proper values of the coupling constant which ensures maintaining the target temperature without large oscillations. ${ }^{58,80,81}$

During water spontaneously filling the channels, the instantaneous positions of the advancing capillary front along the direction of the water flow are tracked to estimate the penetration length of the liquid as a function of time (cf. figure 4). The initial capillary front velocity $\left(u_{i}\right)$, the duration of the inertial time $\left(t_{i}\right)$ and the dynamic contact angles for each simulation during nanocapillary imbibition are computed from the atomic trajectories. The physical mechanisms behind the dynamics of water filling a nanochannel with an applied electric field can be rather complex owing to coupled factors such as the water molecules-electric field interactions, the intermolecular forces between species in the system and the variable population of hydrogen bonds in water. ${ }^{47,82}$ Therefore, to evaluate the effect of an applied electric field on the contact angle of the advancing capillary front, we compute the dynamic contact angle (DCA) during water imbition in the silica nanochannel (cf. figure 5). For details about extracting DCA values during the water imbibition, the readers are referred to our previous study. ${ }^{45}$

We observe that the dynamic contact angles measured during the capillary imbibition in either the absence or presence of externally applied fields (cf. figure 5) are consistent with the values computed of equilibrium WCA of nanodroplets as shown in figure 2 . For instance, figure 5 shows that the DCA value for the case without applied field (black squares) evolves from an initial value of ca. $84^{\circ}$ until attaining a constant value of ca. $21^{\circ}$ while for the case with the highest applied field (blue triangles), the DCA evolves from the same initial value until attaining a constant value of ca. $13^{\circ}$, similar to the corresponding equilibrium WCA values shown in figure 2 . Therefore, our results reveal a substantial decrease in both the dynamic and static contact angles as higher electric field are applied suggesting a significant field-dependent increase in the Laplace pressure at the capillary meniscus.

In figure 4, we plot temporal evolution of the imbibition length for different electric field strengths. The time evolution of the imbibition lengths during the initial stage of the filling is linear irrespective of the strength of the imposed electric field which indicates a capillary flow with constant velocity at the channel entrance confirming, for all the cases, the existence of an inertial regime as predicted by Bosanquet. ${ }^{41,42,45,83,84}$ This insensitivity of the meniscus speed to the particular strength of the imposed electric field implies that the initial contact angle (ca. $80^{\circ}$ ) at the channel entrance does not depend upon the strength of the applied field and should remain constant during the inertial regime, in line with the results in our previous study ${ }^{45}$. Nevertheless, figure 4 also indicate that the inertial time ${ }^{42}$, during which retarding inertial forces dominate capillary filling, decreases with increasing strength of the applied field. These shorter transition times suggest that properties in the imbibed water vary systematically with the strength of the applied electric field.

Once the flow transitions from the inertial to the viscous regime, the results clearly reveal that water filling rates decrease for higher magnitudes of the applied field in channels with the same height. We observe a difference of $40 \%$ in the maximum imbibition velocity, measured at the center of the channel, during the viscous regime for the two extreme cases i.e. the case without applied electric field and the one with the maximum applied field of $1.0 \mathrm{~V} / \mathrm{nm}$ (See Supplementary information). It should be noticed that a capillary meniscus with lower contact angle would imply a higher Laplace pressure ${ }^{83,85}$ and thus, a higher filling speed. Therefore, a decrease in filling rates (figure 4) is in contradiction with the observed lowering of contact angle under increasing electric fields as shown in figures 2 and 5 .

Another important aspect related to capillary imbibition is the surface tension of the imbibing liquid which for water, is closely related to the number of interfacial hydrogen bonds and has been reported in detail elsewhere. ${ }^{49,86,87}$ Indeed, in presence of external electric fields, changes in the meniscus surface tension could potentially alter the Laplace pressure during capillary filling ${ }^{88}$ and consequently modify the flow rates in the channel. Recently, the surface tension of water has been reported to increase within the range of electric field strengths under study 


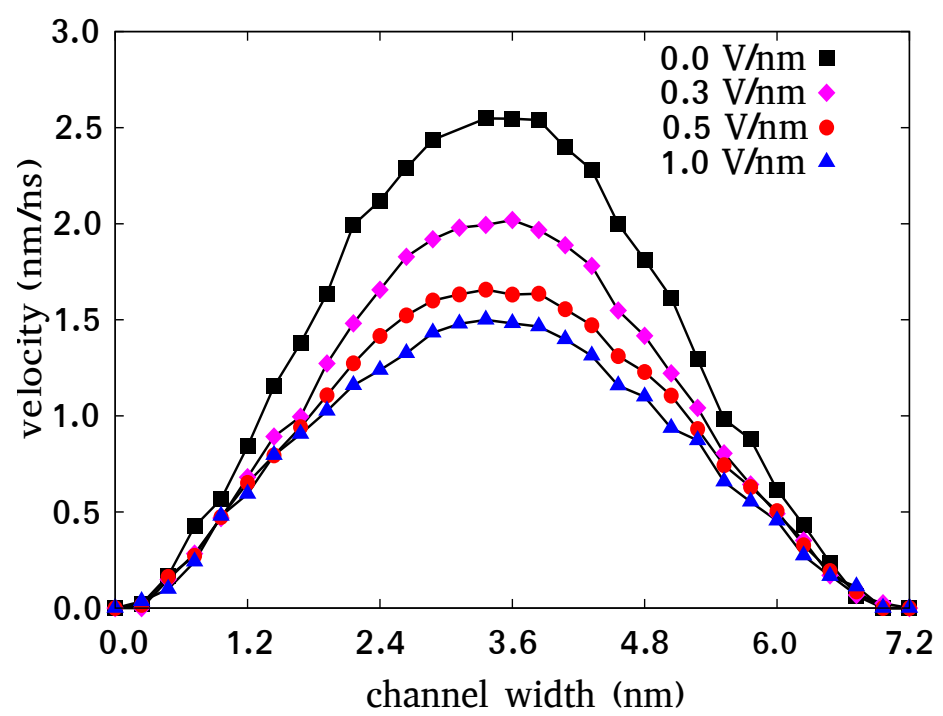

Fig. 6 Velocity profile of Poiseuille flow for a slit silica channel with height of $7 \mathrm{~nm}$ at different applied field strengths. The maximum velocity computed at the center of the channel decreases with increasing applied field strength.

here. ${ }^{87}$ Therefore, indicating that the effect of the applied electric field on water surface tension is not an explanation of the decrease in filling rates observed in the present study.

\section{Simulations of Poiseuille like flow in silica nanochan- nels}

To understand the causes of this apparent contradiction, we performed further MD simulations of Poiseuille like flow in silica nanochannels with height of $7 \mathrm{~nm}$. Considering water flow sufficiently far from the channel entrance and also far behind of the capillary front position is a scenario wherein it is acceptable to assume Poiseuille flow to study the influence of an electric field on viscosity during capillary filling. All the conditions during the study of Poiseuille like flow are maintained constant except for the strength of the axial electric field in the flow direction. We perform simulations at various field strengths viz. 0.0, $0.3,0.5,0.7$ and $1.0 \mathrm{~V} / \mathrm{nm}$. We equilibrate the water for $3 \mathrm{~ns}$ in NVT and $3 \mathrm{~ns}$ in NVE ensembles, respectively. After water is equilibrated, a constant acceleration (gravity field) is imposed to each water molecule to reproduce a Poiseuille like flow through the silica channel. In order to extract the viscous heat, a Berendsen thermostat ${ }^{76}$ with a coupling time constant of $0.1 \mathrm{ps}$ at $300 \mathrm{~K}$ is connected to the water molecules. For additional details about the setup of the Poiseuille flow simulations, the readers are referred to Wagemann et al. ${ }^{63}$ The production simulations are performed for $20 \mathrm{~ns}$ with an electric field applied in the flow direction. In all the simulations with Poiseuille

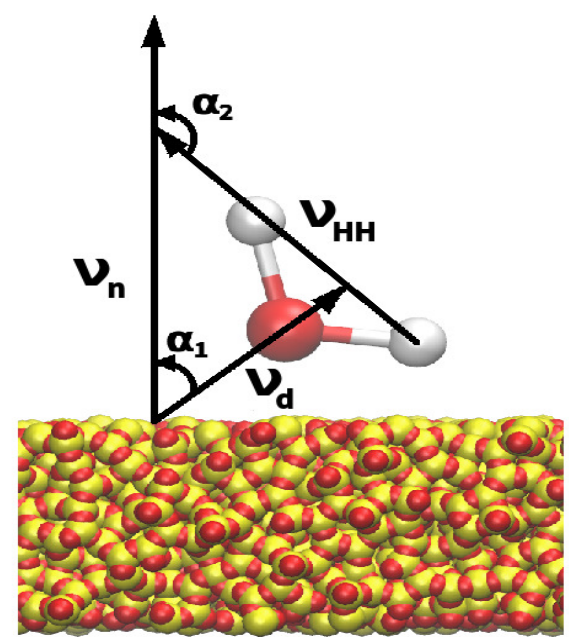

Fig. 7 The angles considered to determine the orientation of the water molecules are given in terms of the angles $\alpha_{1}$ and $\alpha_{2}$. $v_{d}, v_{n}$ and $v_{H H}$ are the vectors corresponding to the dipole moment, normal to the surface and that connecting the two hydrogen atoms, respectively.

like flow, a gravity field is applied to impose an acceleration of $9.83 \cdot 10^{11} \mathrm{~m} / \mathrm{s}^{2}$, equivalent to a pressure gradient $10 \mathrm{bar} / \mathrm{nm}$. Velocities are computed by averaging over the axial length after steady state velocity profiles have been reached, more details regarded the methodology can be found in our previous studies. ${ }^{63}$

The velocity profiles across the channel at various field strengths shown in figure 6 indicate that the maximum velocity decreases with increase in the strength of the imposed electric field. These results confirm our previous observations of reduced capillary filling rates with higher applied electric fields. We note that the $\sim 20 \%$ increase in bulk viscosity at $1 \mathrm{~V} / \mathrm{nm}$ reported in the previous section cannot account for the ca. $40 \%$ decrease in maximum velocity observed for the cases with Poiseuille flow (See supporting information $\mathrm{S} 1$ for the plot of maximum velocity observed during visco-inertial regimes at different electric fields).

Orientation of water molecules under the influence of an external electric field

Now that we have discussed the effect on imbition of viscosity and surface tension under an externally applied electric field, we turn to the effect of electric field inducing water orientation and its influence on the observed lower than expected nanocapillary imbibition. To shed light into the problem, we measure the orientation of water molecules under nanoconfinement. The orientation profiles, describing water angle distribution, are defined by two specific an- 


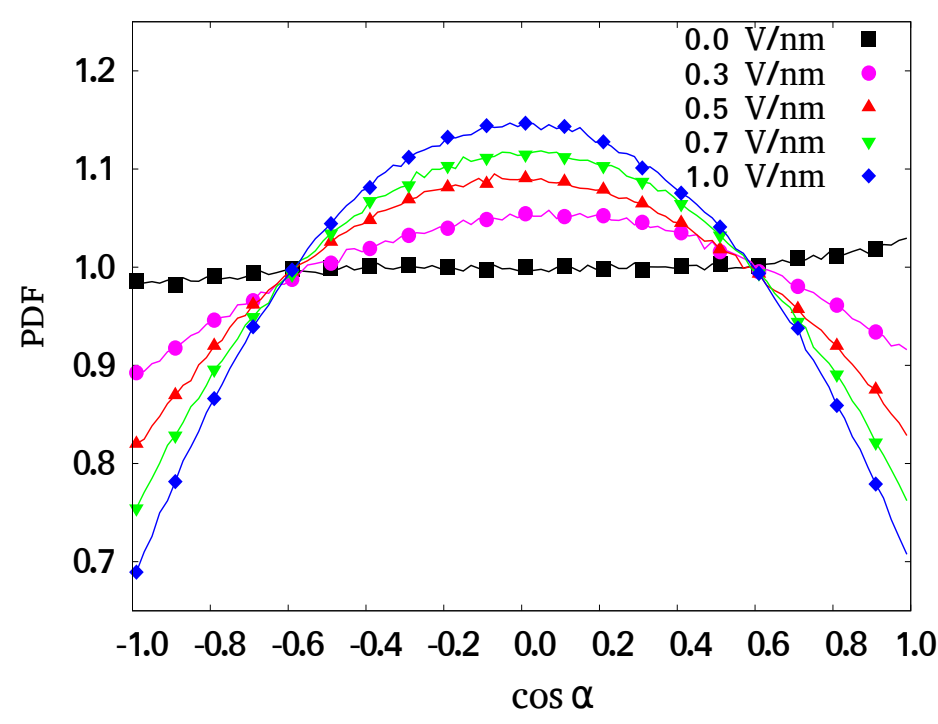

Fig. 8 Probability distribution function of water dipole moment at a distance between 1.5-2.0 nm from the lower wall surface.

gles: $\alpha_{1}$ and $\alpha_{2}$ as illustrated in figure $7, \alpha_{1}$ is the angle between the dipole vector $\left(v_{d}\right)$ of a single water molecule and the normal vector $\left(v_{n}\right)$ for the surface, while $\alpha_{2}$ is the angle between $\left(v_{H H}\right)$, the vector from one $\mathrm{H}$ atom to the other in the same water molecule, and $\left(v_{n}\right)$. The preferred orientation of water molecules under external electric fields has been studied extensively in different systems. ${ }^{38,47,51,89}$ Specifically, the response of nanoconfined water molecules to an externally applied electric field can be inferred from the orientation of the water dipole moment (cf. figure 8). In this figure, the probability distribution function (PDF) of the angle between the vector normal to the silica surface and the water dipole has been calculated at the distance between 1.5 and $2.0 \mathrm{~nm}$ from the silica surface. We choose this distance as, for pure water, the effect of a solid surface does not significantly affect the water density beyond $1.5 \mathrm{~nm}$ (cf. figure 9) and hence, is considered to have bulk properties beyond this distance. ${ }^{90,91}$ In fact, as shown in Supporting information (Figure S2 and S4), we observed a high preferential orientation of water molecules near the channel walls for all the cases with and without applied electric fields, in line with previous experimental studies. ${ }^{91}$ However, the molecular orientation quickly vanishes in the bulk in absence of an external electric field (see figure S2 of the Supporting information). Conversely, in the presence of electric fields (see figure 8 and figure S3 of the Supporting information), we observe that the water molecules in the bulk orient themselves along the field with the oxygen atoms against, and the hydrogen, along the field direction. ${ }^{38,49,51}$ Moreover, in the bulk, the water molecules for the highest strength of applied field display a preferential orientation of $80^{\circ}-110^{\circ}$ indicating that the dipole moment is nearly tangential to the channel wall surface.

To further study the structure of the water molecules in the channel, we consider the orientation of the vector that connects the two hydrogen atoms $\left(v_{H H}\right.$ in figure 7) which in other words indicates the particular plane wherein water molecules are localized. Our results show that the plane of water molecules tends to be oriented perpendicular to the wall surface with increase in the applied electric field strength (see Supporting information S3). The position of the plane is indicated by the increased probability of orientation of the vector $v_{H H}$ which points perpendicular to the surface wall. This preferential orientation suggests that the water molecules are highly structured with oxygen atoms oriented against the applied field with one $\mathrm{OH}$ bond pointing towards the surface and other towards the bulk. Nevertheless, we infer that the interaction with the partial charges of surface silicon and oxygen atoms leads to water molecules with suppressed translational and rotational freedom near channel walls which results in a slowdown of the interfacial water dynamics. ${ }^{92}$ In accordance with this, the distribution function of water dipole angle with the normal to the wall surface reveals that the water molecules in the bulk are more prone to respond to the particular strength of the applied field ${ }^{51}$ (cf. figure 8) compared to water molecules near the wall surface (See supporting information S4). This differential response of water molecules across the channel to externally applied electric fields can affect the overall hydrogen bond distribution in the system and thus resulting in interfacial water with a fluidity intrinsically distinct than that in bulk water. ${ }^{24,25,72,90,93}$ Furthermore, highly structured water at the solid-liquid interface ${ }^{91}$ might result in an increased friction coefficient. ${ }^{23,94}$ Therefore, an altered fluidity of the interfacial water ${ }^{95,96}$ combined with an increase in the viscosity in bulk may govern hydrodynamics during water capillary filling of nanochannels under external electric fields.

To estimate the mobility of water under these conditions, we measure the self-diffusion coefficient of water along the channel cross section at different electric field strengths (cf. figure 9). We calculate the three components of the selfdiffusion coefficient in the box viz. $D_{x}, D_{y}$ and $D_{z}$ representing $x, y$, and $z$ components respectively, which are calculated from the asymptotic slope of the time-dependent mean-square displacement (MSD). However, we assume the effective self-diffusion of the fluid in the direction parallel to the electric field as $D_{e f f}=D_{x}$. We consider only the $x$-component of the self-diffusion coefficient taking into account the direction of the capillary flow. We find that the self-diffusion coefficient of water is affected by the presence of walls and decreases at the wall-fluid interface (cf. figure 9). The self-diffusion coefficient of water can be re- 
Table 1 Parameters of equation 6 under different magnitudes of electric field at $300 \mathrm{~K}$

\begin{tabular}{llll}
\hline E-field $(\mathrm{V} / \mathrm{nm})$ & $\frac{D_{b u l k}}{D_{a p p}}$ & $\frac{\mu_{e f f}}{\mu_{b u l k}}$ & Contribution(\%) to the effective viscosity \\
\hline 0.0 & 1.66 & 1.10 & $10 \%$ \\
0.5 & 1.41 & 1.06 & $6 \%$ \\
1.0 & 1.30 & 1.05 & $5 \%$ \\
\hline
\end{tabular}

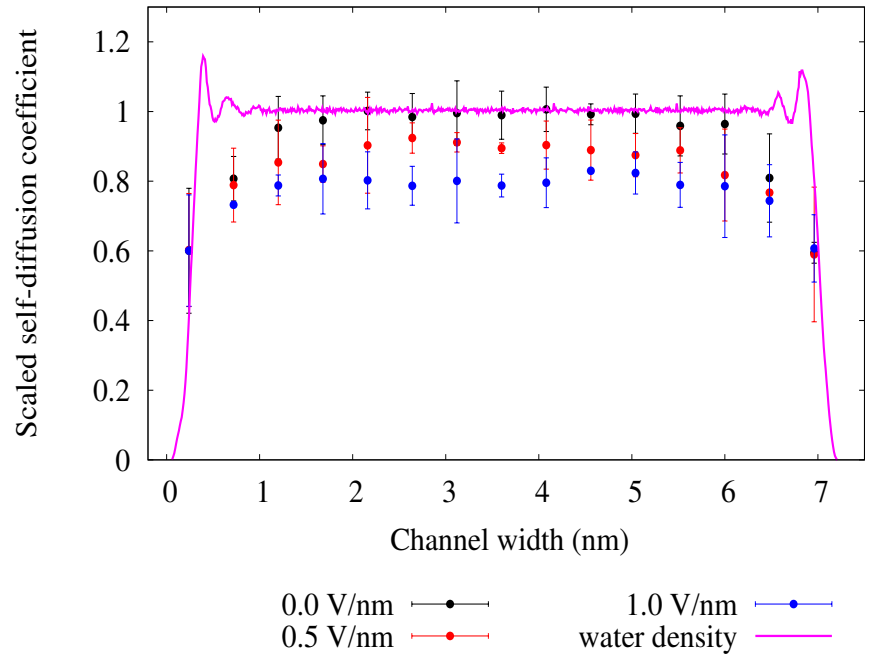

Fig. 9 Coefficient of self-diffusion of water along channel width. The solid line represents the density of water and the dots with error bars represent the scaled self-diffusion coefficient of water calculated using Stokes-Einstein relation.

lated to the viscosity using Stokes-Einstein relation ${ }^{51,97}$ as shown in equation 5 .

$$
\mu_{e f f}=\frac{k_{B} T}{3 \pi r_{w}} \frac{1}{D_{e f f}}
$$

$\mu_{e f f}$ is the effective viscosity of water, where $r_{w}$ is the effective molecular diameter which is a function of the size and shape of the molecule (for water molecules, $r_{w}=$ $0.17 \mathrm{~nm}^{98,99}$ ), and $D_{\text {eff }}$ is the self-diffusion coefficient. Moreover, using the Einstein's relation (equation 5), it can be inferred that the viscosity of water is higher at the wallwater interface compared to the viscosity in the bulk. We observe that the change in interfacial viscosity is less than changes reported in other studies ${ }^{100}$ and that the contribution of the interfacial viscosity to the effective viscosity is relatively low and decreases with an increase in electric field strength. Indeed, we calculate the contribution of interfacial viscosity to the total effective viscosity using height dependent viscosity of water in nano-conduits ${ }^{100}$ as shown in equation 6

$$
\frac{\mu_{e f f}}{\mu_{b u l k}}=1+\frac{2 L_{i}}{H}\left(\frac{\mu_{a p p}}{\mu_{b u l k}}-1\right)
$$

where $\mu_{\text {bulk }}$ is the viscosity of the bulk, and $\mu_{a p p}$ is the interfacial viscosity. $L_{i}$ represents the width of the interface while $H$ is the height of the channel. In this study, we consider interface to be with $0.6 \mathrm{~nm}$ from the channel walls, where maximum water density is found. For the magnitudes of electric field under study, the values of the parameters in equation 6 are listed in table 1 . We noticed that this increase in total effective viscosity due to contribution of the higher interfacial viscosity still cannot explain the lowering of maximum velocity observed during the capillary imbibition under the influence of electric field. All the above results suggest the contribution of an additional interfacial phenomenon that slows down the filling rate of water in the hydrophilic nanochannels.

\section{Influence of electric field on solid-liquid interfacial fric- tion}

The increased ordering and lower mobility of water at the interface suggests a higher friction at the walls as compared to systems without external electric fields. To further shed light into this, we calculated the interfacial friction coefficient $(\lambda)$ near the silica walls in a parallel silica channel with water confined at $300 \mathrm{~K}$. According to linear response theory, $\lambda$ can be obtained from the equilibrium fluctuations of the friction force, using a Green-Kubo (GK) relation ${ }^{94,101}$

$$
\lambda=\lim _{t \rightarrow \infty} \lambda_{G K}(t)
$$

with

$$
\lambda_{G K}(t)=\frac{1}{2 \mathscr{A} k_{B} T} \int_{0}^{t}\left\langle F\left(t^{\prime}\right) \cdot F(0)\right\rangle d t^{\prime}
$$

where, $\mathscr{A}$ is the surface area of the wall, $T$ is the temperature, and $F(t)$ is the total tangential force acting along the $x$-axis at the liquid/solid interface and the factor of $1 / 2$ comes from the averaging over the two spatial dimensions parallel to the sheets. The friction coefficients calculated using GK relation for sufficiently long time plateaus to a constant value of $\lambda_{G K}$. We obtain the value of $\lambda_{G K}$ from the plateau of the integrated force autocorrelation function in the range of $0-0.15 \mathrm{ps}$. We take this plateau value as the equilibrium value for the friction coefficient following the methodology presented by Falk et al. ${ }^{102}$. We plot these cal- 


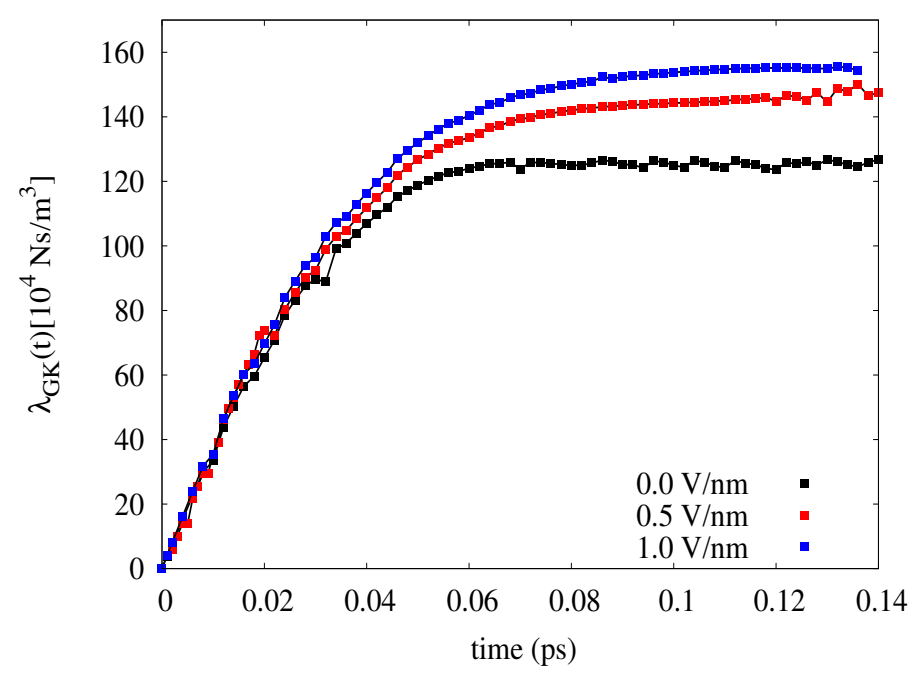

Fig. 10 Interfacial friction coefficient in water-silica wall interface.

culated values of the friction coefficients for three different cases in figure 10. We observe a significant increase in the calculated values of friction coefficients with increase in electric field in the direction parallel to the silica surface. For the case with applied electric field of $1.0 \mathrm{~V} / \mathrm{nm}$, we find approximately ca. $33 \%$ increase in $\lambda_{G K}$, compared to the case with no applied field.

\section{Conclusions}

In summary, we have presented a study on the effect of externally applied electric fields on capillary flow of water in amorphous silica nanochannels. We compute lower equilibrium DCAs of the capillary meniscus as electric fields with increasing strengths are applied. Furthermore, we find that the overall filling rates decrease with increasing electric field strengths. We attribute this to an increase in effective viscosity due to the alignment of water molecules in the bulk and also to the particular structure of the interfacial water molecules under combined effect of the silica wall surface and the presence of the external electric field. Our results indicate that the ordering induced by the electric field results in an altered solid-liquid friction which combined to a substantial increase in bulk viscosity dominate the capillary force balance during water imbibition. In particular, our study suggests that the ordering of water molecules affects the "propagation" of momentum dissipation between the solid surface and liquid water such that the friction is readily transferred to the bulk in highly oriented water molecules. This finding from our atomistic simulations enriches the general discussions of the solidliquid interface related phenomena and its critical effect in transport of fluid in hydrophilic nanochannels. Moreover, our results are helpful for a comprehensive understanding of the complex phenomenon of water capillary filling of nanopores under the effect of externally applied electric fields.

\section{Conflict of interest}

There are no conflicts of interest to declare.

\section{ACKNOWLEDGEMENT}

This research was partially funded by CONICYT under scholarship No 21140427 and FONDECYT project no. 11130559. The authors thank computational support from Danish Center for Scientific Computing (DCSC) and from National Laboratory for High Performance Computing (NLHPC) Chile. E. Wagemann thanks financial support from Centro CRHIAM Conicyt/Fondap Project 15130015.

\section{References}

1 H. Cabral, N. Nishiyama and K. Kataoka, Accounts of Chemical Research, 2011 44, 999-1008.

2 L. Li, Y. Kazoe, K. Mawatari, Y. Sugii and T. Kitamori, J. Phys. Chem. Lett., 2012, 3, 2447-2452.

3 J. Haneveld, N. Tas, N. Brunets, H. Jansen and M. Elwenspoek, J. Appl. Phys., 2008, 104, 014309.

4 S. Gravelle, L. Joly, F. Detcheverry, C. Ybert, C. Cottin-Bizonne and L. Bocquet, Proc. Natl. Acad. Sci. USA, 2013, 110, 16367.

5 C. Wu, T. G. Lin, Z. Zhan, Y. Li, S. C. Tung and W. J. Li, Microsystems Amp Nanoengineering, 2017, 3, 1-8.

6 M. Ferrari, Nature Rev. Cancer, 2005, 5, 161-171.

7 V. Okhonin, X. Liu and S. N. Krylov, Anal. Chem., 2005, 77, 5925-5929.

8 Y. Temiz, R. D. Lovchik, G. V. Kaigala and E. Delamarche, Microelectronic Engineering, 2015, 132, $156-175$.

9 J. Chen, C. Xue, Y. Zhao, D. Chen, M.-H. Wu and J. Wang, International Journal of Molecular Sciences, 2015, 16, 9804-9830.

10 P. M. Sinha, G. Valco, S. Sharma, X. Liu and M. Ferrari, Nanotechnology, 2004 15, S585.

11 S. M. Park, Y. S. Huh, H. G. Craighead and D. Erickson, Proc. Natl. Acad. Sci. USA, 2009, 106, 15549-15554.

12 M. Henderson, Surf. Sci. Rep., 2002, 46, 1-308.

13 C.-C. Lai, C.-J. Chang, Y.-S. Huang, W.-C. Chang, F.-G. Tseng and Y.-L. Chueh, Nano Energy, 2015, 12, 394 - 400.

14 B. Wolfrum, E. Katelhon, A. Yakushenko, K. J. Krause, N. Adly, M. Huske and P. Rinklin, Accounts of Chemical Research, 2016, 49, 2031-2040.

15 J. Lee, T. Laoui and R. Karnik, Nature Nanotechnol., 2014, 9, 1-7.

16 Q. Xie, M. A. Alibakhshi, S. Jiao, Z. Xu, M. Hempel, J. Kong, H. G. Park and C. Duan, Nature, 2018, 13, 238-246.

17 S. Chakraborty, H. Kumar, C. Dasgupta and P. K. Maiti, Acc. Chem. Res., 2017, 50, 2139-2146.

18 N. E. Levinger, Science, 2002, 298, 1722-1723.

19 J. Kofinger, G. Hummer and C. Dellago, Proc. Natl. Acad. Sci. USA, 2008, 105, 13218-13222.

20 C. Lee, C. Cottin-Bizonne, R. Fulcrand, L. Joly and C. Ybert, The Journal of Physical Chemistry Letters, 2017, 8, 478-483.

21 K. Wu, Z. Chen, J. Li, X. Li, J. Xu and X. Dong, Proc. Natl. Acad. Sci. USA, 2017, 114, 3358-3363.

22 D. Ortiz-Young, H.-C. Chiu, S. Kim, K. Voitchovsky and E. Riedo, Nat. Commun., 2013, 4, 1-6.

23 X. Xia and M. L. Berkowitz, Phys. Rev. Lett., 1995, 74, 3193-3196.

24 Z. Qian, Z. Fu and G. Wei, J. Comput. Phys., 2014, 140, 1-5.

25 A. Vegiri and S. V. Schevkunov, J. Chem. Phys., 2001, 115, 4175-4185.

26 W. J. Rieter, K. M. L. Taylor and W. Lin, Journal of the American Chemical Society, 2007, 129, 9852-9853.

27 S. Prakash, T. M. Long, J. C. Selby, J. S. Moore and M. A. Shannon, Anal. Chem., 2007, 79, 1601-1667.

28 Y. Xue, J. Markmann, H. Duan, J. Weissmuller and P. Huber, Nat. Commun., 2014, 5, 1-8.

29 H. A. Zambrano, N. Vasquez and E. Wagemann, Phys. Chem. Chem. Phys., 2016, 18, 1202-1211.

30 M. Fuest, C. Boone, K. K. Rangharajan, A. T. Conlisk and S. Prakash, Nano Letters, 2015, 15, 2365-2371.

31 A. Schultz, I. Papautsky and J. Heikenfeld, Langmuir, 2014, 30, 5349-5356.

32 K. Xiao, Y. Zhou, X.-Y. Kong, G. Xie, P. Li, Z. Zhang, L. Wen and L. Jiang, ACS Nano, 2016, 10, 9703-9709.

33 M. Sadeghi-Goughari, S. Jeon and H.-J. Kwon, Physics Letters A, 2017, 381, 2898-2905. 
34 C. Bakli and S. Chakraborty, Nanoscale, 2016, 8, 6535-6541.

35 L. Chen and A. T. Conlisk, Biom. Microdevices, 2008, 10, 289-298.

36 S. McLaughlin and R. T. Mathias, J. Gen. Physiol., 1985, 85, 699-728.

37 I. S. Davis, B. Shachar-Hill, M. R. Curry, K. S. Kim, T. J. Pedley and A. E. Hill, Proc. R. Soc. A, 2007, 463, 881-896.

38 K. Ritos, M. K. Borg, N. J. Mottram and J. M. Reese, Philosophical Transactions of the Royal Society A Mathematical Physical and Engineering Sciences, 2016, $374,1-5$.

39 D. I. Dimitrov, A. Milchev and K. Binder, Phys. Chem. Chem. Phys., 2008, 10, 1867-1869.

40 P. S. Laplace, Courcier Paris, 1805, 2 Supplement au Livre X, 349-498.

41 C. H. Bosanquet, Phil. Mag. Ser. 6, 1923, 45, 525-531.

42 E. Oyarzua, J. H. Walther, A. Mejia and Z. H. A., Phys. Chem. Chem. Phys., 2015, 17, 14731-14739.

43 E. R. Cruz-Chu, A. Aksimentiev and K. Schulten, J. Phys. Chem. B, 2006, 110, 21497-21508.

44 N. Giovambattista, P. G. Debenedetti and P. Rossky, J. Phys. Chem. B, 2007, 111, 9581-9587.

45 N. K. Karna, E. Oyarzua, J. H. Walther and H. A. Zambrano, Phys. Chem. Chem. Phys., 2016, 18, 31997-32001.

46 T. Krupenkin and J. A. Taylor, Nature, 2011, 2, 448.

47 C. D. Daub, D. Bratko, K. Leung and A. Luzar, The Journal of Physical Chemistry C, 2007, 111, 505-509.

48 F. H. Song, B. Q. Li and C. Liu, Langmuir, 2013, 29, 4266-4274.

49 F. H. Song, B. Q. Li and Y. Li, Phys. Chem. Chem. Phys., 2015, 17, 5543-5546.

50 Winarto, D. Takaiwa, E. Yamamoto and K. Yasuoka, The Journal of Chemical Physics, 2015, 142, 124701.

51 D. Zong, H. Hu, Y. Duan and Y. Sun, The Journal of Physical Chemistry B, 2016, 120, 4818-4827.

52 I.-C. Yeh and M. L. Berkowitz, The Journal of Chemical Physics, 1999, 110, 7935-7942.

53 V. B. Warshavsky and X. C. Zeng, Phys. Rev. Lett., 2002, 89, 246104.

54 Z. Wang, D. L. Olmsted, M. Asta and B. B. Laird, Journal of Physics: Condensed Matter, 2016, 28, 464006.

55 Y. Yang, C. Thompson, V. Anderson and A. Wood, Physica A: Statistical Mechanics and its Applications, 1999, 268, $424-432$.

56 Q.-N. Zheng, X.-H. Liu, X.-R. Liu, T. Chen, H.-J. Yan, Y.-W. Zhong, D. Wang and L.-J. Wan, Angewandte Chemie International Edition, 2014, 53, 13395-13399.

57 T.-H. Yen, $M S, 2012,38,509-517$.

58 N. J. English and C. J. Waldron, Phys. Chem. Chem. Phys., 2015, 17, 1240712440 .

59 H. Frolich, in Theory of dielectrics dielectric constant and dielectric loss, Oxford University Press, 1990, pp. 25-30.

60 A. M. Saitta, F. Saija and P. V. Giaquinta, Phys. Rev. Lett., 2012, 108, 207801.

61 A. T. Celebi, M. Barisik and A. Beskok, J. Comput. Phys., 2017, 147, 1-10.

62 I. M. Svishchev and P. G. Kusalik, Phys. Rev. Lett., 1994, 73, 975-979.

63 E. Wagemann, E. Oyarzua, J. H. Walther and H. A. Zambrano, Phys. Chem. Chem. Phys., 2017, 19, 8646-8652.

64 J. H. Walther, R. Jaffe, T. Halicioglu and P. Koumoutsakos, J. Phys. Chem. B, 2001, 105, 9980-9987.

65 E. Oyarzua, J. H. Walther, C. M. Megaridis, P. Koumoutsakos and H. A. Zambrano, ACS Nano., 2017, 11, 9997-10002.

66 H. J. C. Berendsen, J. R. Grigera and T. P. Straatsma, J. Phys. Chem., 1987, 91, 6269-6271.

67 Y. Guissani and B. Guillot, J. Chem. Phys., 1996, 104, 7633-7644.

68 S. Tsuneyuki, M. Tsukada, H. Aoki and Y. Matsui, Phys. Rev. Lett., 1988, 61, 869-874.

69 H. A. Zambrano, J. H. Walther and R. L. Jaffe, J. Mol. Liq., 2014, 107 - 113.

70 L. H. Thamdrup, F. Persson, H. Bruus, A. Kristensen and H. Flyvbjerg, Appl. Phys. Lett., 2007, 91, 163505.

71 J. Schmidt, S. Roberts, J. Loparo, A. Tokmakoff, M. Fayer and J. Skinner, Chemical Physics, 2007, 341, $143-157$.

72 S. Romero-Vargas, N. Giovambattista, I. A. Aksay and P. G. Debenedetti, J. Phys. Chem. B, 2009, 113, 1438-1446.

73 F. Romer, A. Lervik and F. Bresme, J. Chem. Phys., 2012, 137, 074503.

74 C. Vega and E. de Miguel, The Journal of Chemical Physics, 2007, 126, 154707.

75 J. H. Weijs, A. Marchand, B. Andreotti, D. Lohse and J. H. Snoeijer, Physics of Fluids, 2011, 23, 022001.

76 H. J. C. Berendsen, J. P. M. Postma, W. F. van Gunsteren, A. DiNola and J. R. Haak, J. Chem. Phys., 1984, 81, 3684-3684.

77 X. Chen, P. Carbone, W. L. Cavalcanti, G. Milano and F. Müller-Plathe, Macromolecules, 2007, 40, 8087-8095.

78 B. Hess, The Journal of Chemical Physics, 2002, 116, 209-217.

79 A. P. Sunda and A. Venkatnathan, Molecular Simulation, 2013, 39, 728-733.

80 R. Reale, N. J. English, P. Marracino, M. Liberti and F. Apollonio, Mol. Phys., 2014, 112, 1870-1878.

81 N. J. English and J. M. D. MacElroy, The Journal of Chemical Physics, 2003, 119, 11806-11813.

82 F. Mugele and J.-C. Baret, Journal of Physics: Condensed Matter, 2005, 17, R705.
83 K. G. Kornev and A. V. Neimark, J. Coll. Interface Sci., 2001, 235, 101-113. 84 S. Das, P. R. Waghmare and S. K. Mitra, Phys. Rev. E, 2012, 86, 067301.

85 D. Yang, M. Krasowska, C. Priest, M. N. Popescu and J. Ralston, J. Phys. Chem. C, 2011, 115, 18761-18769.

86 I. V. Stiopkin, C. Weeraman, P. A. Pieniazek, F. Y. Shalhout, J. L. Skinner and A. V. Benderskii, Nature, 2005, 474, 192-195.

87 M. Nikzad, A. R. Azimian, M. Rezaei and S. Nikzad, J. Chem. Phys., 2017, 147, 204701.

88 A. Bateni, A. Amirfazli and A. W. Neumann, Colloids and Surfaces A: Physicochem. Eng. Aspects, 2006, 289, 25-38.

89 D. Zong, Z. Yang and Y. Duan, Applied Thermal Engineering, 2017, 122, $71-$ 79.

90 O. Bjorneholm, M. H. Hansen, A. Hodgson, L.-M. Liu, D. T. Limmer A. Michaelides, P. Pedevilla, J. Rossmeisl, H. Shen, G. Tocci, E. Tyrode, M.-M. Walz, J. Werner and H. Bluhm, Chemical Reviews., 2016, 116, 7698-7726.

91 P.-A. Mante, C.-C. Chen, Y.-C. Wen, H.-Y. Chen, S.-C. Yang, Y.-R. Huang, I. Ju Chen, Y.-W. Chen, V. Gusev, M.-J. Chen, J.-L. Kuo, J.-K. Sheu and C.-K. Sun, Scientific Reports, 2014, 4, 1-6.

92 M. F. Harrach, F. Klameth, B. Drossel and M. Vogel, J. Chem. Phys., 2015, 142, 034703.

93 G. Stirnemann, S. Romero-Vargas Castrillon, J. T. Hynes, P. J. Rossky, P. G. Debenedetti and D. Laage, Phys. Chem. Chem. Phys., 2011, 13, 911-917.

94 G. Tocci, L. Joly and A. Michaelides, Nano Letters, 2014, 14, 6872-6877.

95 S. Gruener, T. Hofmann, D. Wallacher, A. V. Kityk and P. Huber, Phys. Rev. E, 2009, 79, 067301.

96 S. Gruener, D. Wallacher, S. Greulich, M. Busch and P. Huber, Phys. Rev. E, 2016, 93, 013102.

97 I.-C. Yeh and G. Hummer, The Journal of Physical Chemistry B, 2004, 108, 15873-15879.

98 Y. Li, F. Wang, H. Liu and H. Wu, Microfluidics and Nanofluidics, 2015, 18, 111-120.

99 T. J. Muller, M. Al-Samman and F. Muller-Plathe, The Journal of Chemical Physics, 2008, 129, 014102.

100 T. Q. Vo and B. Kim, Scientific Reports, 2016, 6, 33881.

101 K. Falk, F. Sedlmeier, L. Joly, R. R. Netz and L. Bocquet, Langmuir, 2012, 28, 14261-14272.

102 K. Falk, F. Sedlmeier, L. Joly, R. R. Netz and L. Bocquet, Nano Letters, 2010, 10 , 4067-4073. 


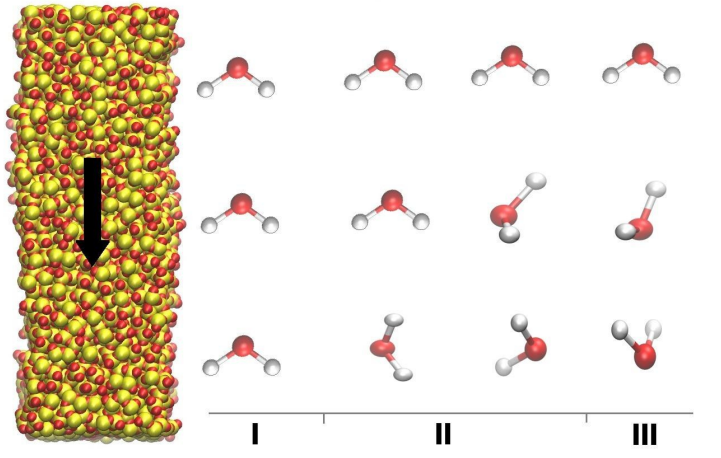

HIGH

MEDIUM

LOW

Graphical abstract. 